GEOPHYSICS, VOL. XXII, NO. 2 (APRII, 1957), PP. 275-285, 10 FIGS.

\title{
A SEISMIC MODEL STUDY OF THE PHASE VELOCITY METHOD OF EXPLORATION*
}

\author{
FRANK PRESS $\dagger$
}

\begin{abstract}
Variations in the phase velocity of earthquake-generated surface waves have been used to determine local variations in the thickness of the earth's crust. It is of interest to determine whether this method can be used to delineate structures encountered by the exploration geophysicist. A seismic model study of the effect of thickness changes, lithology changes; faults-and-scarps; on the phase velocity of surface waves was carried out. It is demonstrated that all of these structures produce measurable variations in the phase velocity of surface waves. Additional information is required, however, to give a unique interpretation of a given phase velocity variation in terms of a particular structure. Some remarks on the phenomenon of returning ground roll are made.
\end{abstract}

\section{INTRODUCTION}

Some of the basic techniques used in exploration seismology stem from methods developed in earthquake seismology. Recently a new method (Press, r 956) for delineating crustal structure by the use of phase velocity variations of earthquake generated surface waves was described. Unlike current practice in seismic exploration where travel times of impulses are determined, this method involves the measurement of local phase velocity of waves of a given frequency. A preliminary evaluation of the applicability of this method in seismic exploration was made using an ultrasonic model analog. In this paper we report on model studies of phase velocity variations due to faults, scarps, thickness changes, and lithology changes.

\section{THE METHOD}

When a disturbance occurs in an elastic half-space in which the elastic constants vary with depth, part of the energy is radiated as body waves and part propagates horizontally, guided by one or more channels associated with particular variations of the elastic parameters. The free surface usually forms an upper boundary to the channel in which case the guided waves are the familiar surface waves. Rayleigh waves, Love waves, Stoneley waves, ground roll, microseisms are examples of guided waves.

Guided waves are dispersed and as a consequence two velocities must be considered, the phase velocity $c$ and the group velocity $U$. The phase velocity is the local velocity of propagation of a point of constant phase such as a trough or peak. It depends on period through an equation which contains as parameters the dimensions of the channel and the elastic constants within and adjacent to the channel. Phase velocity is sensitive to lateral variations in elastic parameters.

\footnotetext{
* Contribution No. 808, Division of Geological Sciences, California Institute of Technology. Presented before the 26th Annual Meeting of the Society in New Orleans on October $3 \mathrm{I}$, 1956. Manuscript received by the Editor December 3, I9.56.

† Seismological Laboratory, California Institute of Technology, Pasadena, California.
} 
For example, when a dispersed train of surface waves crosses a boundary, such as a fault, between two media having different vertical velocity distributions, the waves assume the phase velocity appropriate to the new medium; refraction occurs at the boundary, the paths of the rays being determined by the phase velocities according to the usual law of refraction. The overall travel time of a group of waves of a given frequency is determined by the group velocities (Bullen, I953, p. I06-107).

Data from a single seismogram are sufficient to construct a curve of group velocity versus frequency. By comparison with theoretical group velocity curves constructed under different assumptions one can infer the average properties of the medium between the source and the seismograph (see for example, Ewing and Press, I956).

To obtain indications of local variations in the vertical distribution of elastic parameters, one must use phase velocity. Phase velocity can be determined together with direction of approach from the differential arrival times of individual crests and troughs recorded at three seismographs arranged in a triangular array.

The linear dimensions of the array must be of the order of a wavelength. Smaller separations do not permit precise determinations of differential times whereas longer separations lead to problems of correlating events at each of the stations. Criteria for determining separation distances have been discussed by Evernden (1953).

The phase velocity method is based on the interpretation of local variations in phase velocity using changes in the vertical distribution of elastic parameters. Actually a unique interpretation is not possible since both thickness changes and velocity changes can produce similar phase velocity changes. However, the use of additional information, such as that derived from geological plausibility can sometimes lead to unique interpretations. Although interpretations can be made by comparing observed and theoretical phase velocity curves, a more direct method, one involving fewer assumptions, is to compare observed phase velocity with experimentally determined standard curves (Press, I956).

\section{PROCEDURE}

The two-dimensional seismic model techniques described by Oliver, Press and Ewing (I954), were used. Since dispersion of surface waves is the same for two-dimensional propagation from a line source and three-dimensional propagation from a point source there is no loss in generality of the results. The fabrication of the models is simple and complexities introduced by refraction of surface waves are eliminated. Further simplification is introduced by the use of flexural waves. These waves are easy to excite and the equation governing their dispersion is amenable to fast computation. Although flexural waves do not exist in the earth, they share with naturally occurring waves the general characteristics of guided waves. Satô (I95I) demonstrated the connection between flexural waves and the $M_{2}$ branch of Rayleigh waves. 
Flexural waves were excited in two-dimensional models of structures analogous to those encountered in the field. The waves were observed along spreads crossing the structure, detector spacing being sufficiently small to permit measurement of the resulting phase velocity variation. Models were fabricated from $\mathrm{I} / \mathrm{r} 6$ inch sheets of plexiglass and brass.

\section{RESULTS}

\section{Thickness Change}

This model consists of a plexiglass strip 90 inches long in which the thickness changes from $\frac{1}{4}$ inch to $\frac{1}{8}$ inch in a distance of $\mathrm{I}$ inch at the midpoint. A spread was placed in the range $41^{\frac{1}{2}}-47^{\frac{1}{2}}$ inches so as to cross the thickness change with detector spacing of $\frac{1}{4}$ inch (Figure $\mathrm{r}$ ).

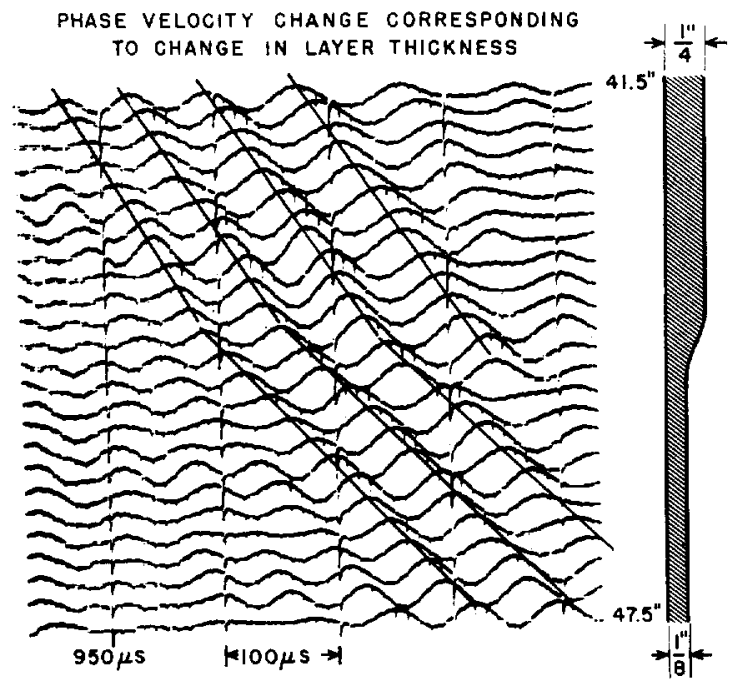

FIG. I. Model seismogram indicating phase velocity change corresponding to thickness change.

Individual crests and troughs may be followed across the structural change, demonstrating the coherence of the waves. Clearly evident is the decrease in phase velocity of the individual waves as they cross from the thick layer to the thin layer. One method of analyzing these data is to use phase velocity for one zone as a standard against which phase velocity data for other zones may be compared. If the propagating medium is one in which structural changes consist only of thickness changes (each layer of the section changing in the same proportion), then the period change for a given phase velocity is proportional to the thickness change. For Figure 2, the experimentally determined phase velocities are plotted as a function of period. The data for the $\frac{1}{4}$ inch zone were extended by computing two points using the flexural wave period equation (Oliver, Press and Ewing, 1954, p. 2 10). Taking the curve for $H=\frac{1}{4}$ inch as a standard, each phase 


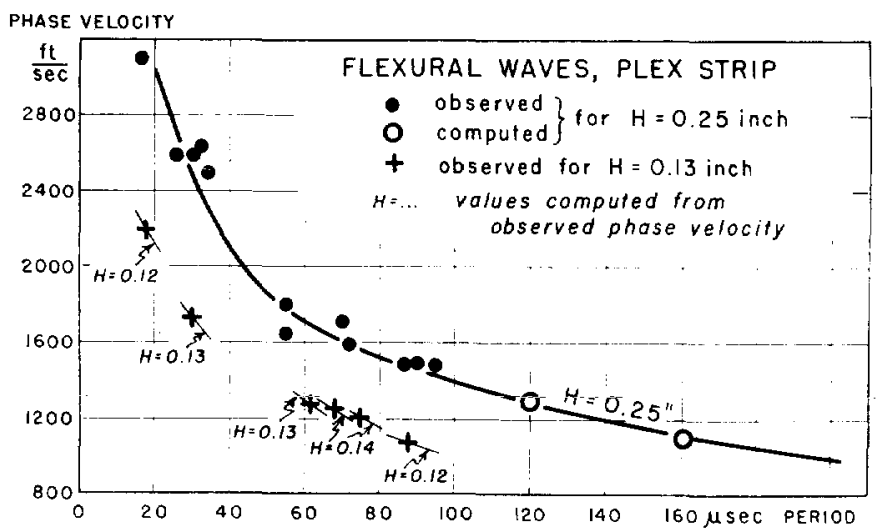

FIG. 2. Graphical interpretation of phase velocity change in terms of thickness change.

velocity observation in the thinner zone was interpreted in terms of a reduced thickness, the computed values being indicated on the figure. The average computed value of 0.13 inch agrees with the actual thickness for this zone.

\section{PHASE VELOCITY CHANGE CORRESPONDING TO CHANGE IN MEDIA}

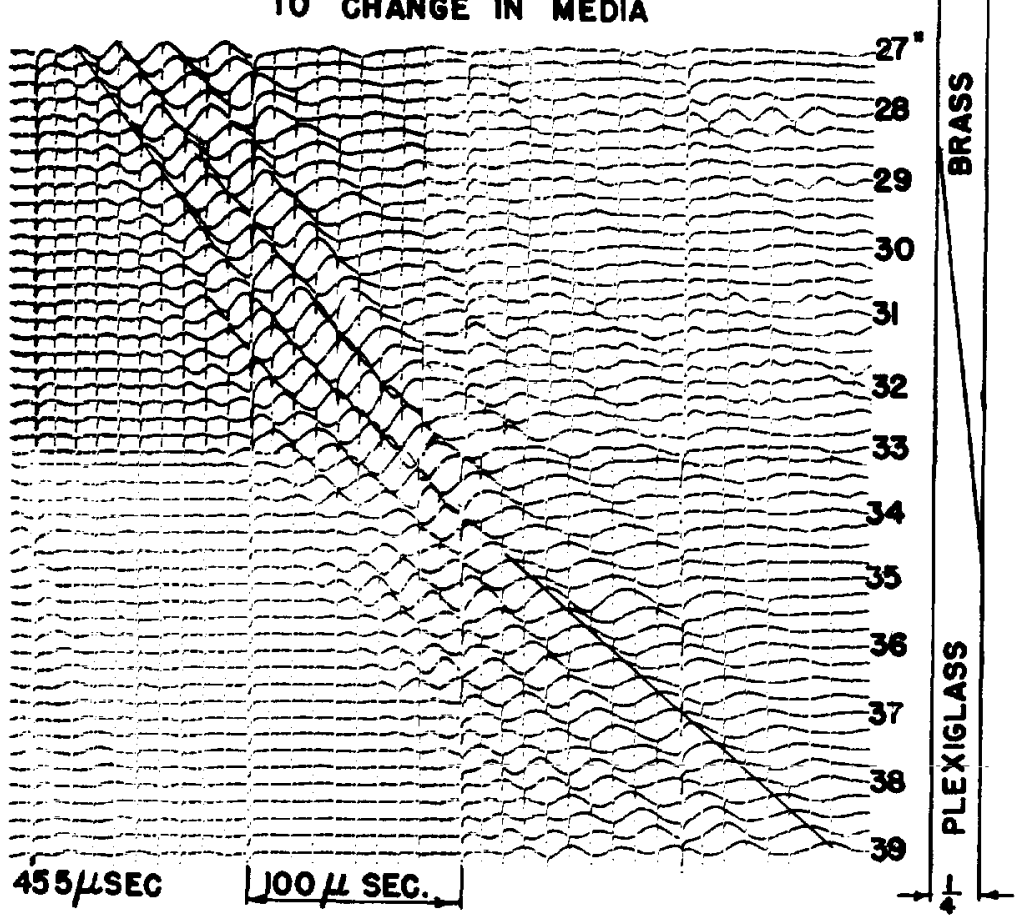

FIG. 3. Model seismogram indicating phase velocity change corresponding to lithology change. 
This analog, simple as it is, demonstrates on the ultrasonic scale the method used by Press (1956) for determining thickness changes of the earth's crust in California.

\section{Lithology Change}

This structure was modeled by a gradual wedgeout from a $\frac{1}{4}$ inch layer of brass to a $\frac{1}{4}$ inch layer of plexiglass over a distance of 6 inches. The impulsive source was located in the brass layer, 32 inches from the midpoint of the wedgeout and observations were made in the range $27-39$ inches (Figure 3 ). Individual crests and troughs may be followe d across the simulated lithology change, the gradual decrease in phase velocity with increasing penetration into the plexiglass zone being evident on the seismogram.

Phase velocities were measured for different periods at several positions along the spread. The results are presented in Figure 4 where the location of the measurement is specified in terms of percent brass in the section. In the same figure curves are presented which were computed from the frequency equation of flex-

\section{PHASE VELOCITY FT./SEC.}

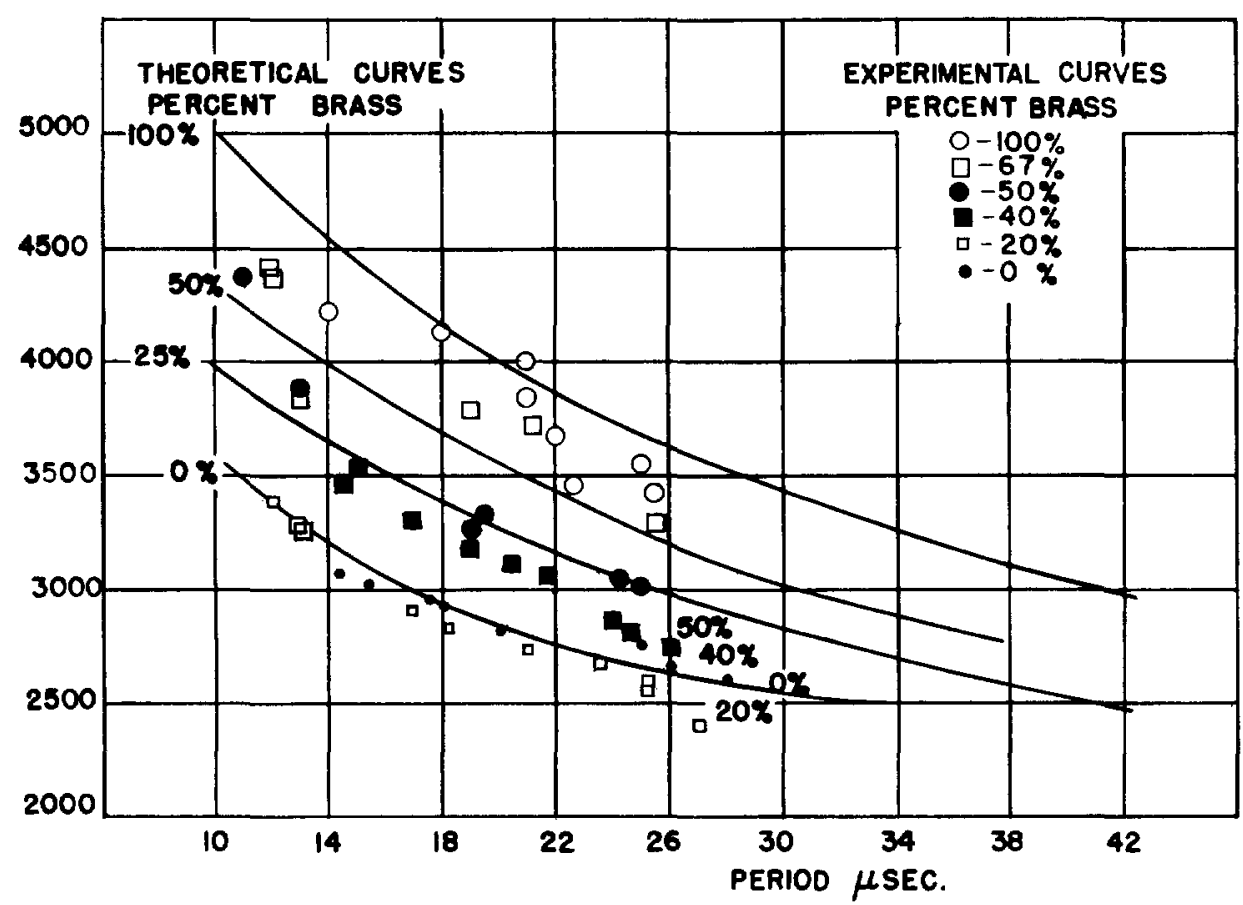

Fig. 4. Comparison of experimental and theoretical phase velocities in a region of lithology change. Theoretical curves based on homogeneous layer with weighted values for elastic constants according to proportion of brass to plexiglass. 
ural waves (Oliver, Press and Ewing, I954) with weighted values for the elastic constants inserted according to the proportion of brass to plexiglass in the sectien. The experimental points for o percent and roo percent brass fit the corresponding computed curves reasonably well. The phase velocity data for the composite sections fall between the pure brass and pure plexiglass curves, but show no systematic agreement with the corresponding computed curves. The lack of systematic agreement between experimental and computed values in the composite zone cannot be interpreted until the theory for flexural waves in a two-layered plate becomes available. Then the validity of phase velocity computations using weighted values for elastic constants can be examined.

This experiment demonstrates how phase velocity variations may be produced by lithology changes. It is not established that composition of a section can be determined in a composite zone, although the occurrence of such a zone may be detectable.

\section{Fault}

The fault is represented by an abrupt change from a brass layer to a plexiglass layer at a distance of $29 \mathrm{I}_{5} / 16$ inches from the source. The detector spread ranges from $23 \frac{3}{4}$ to 36 inches (Figure 5). Indicated on the seismogram are incident flexural waves, waves transmitted across the fault and waves reflected by the fault. The

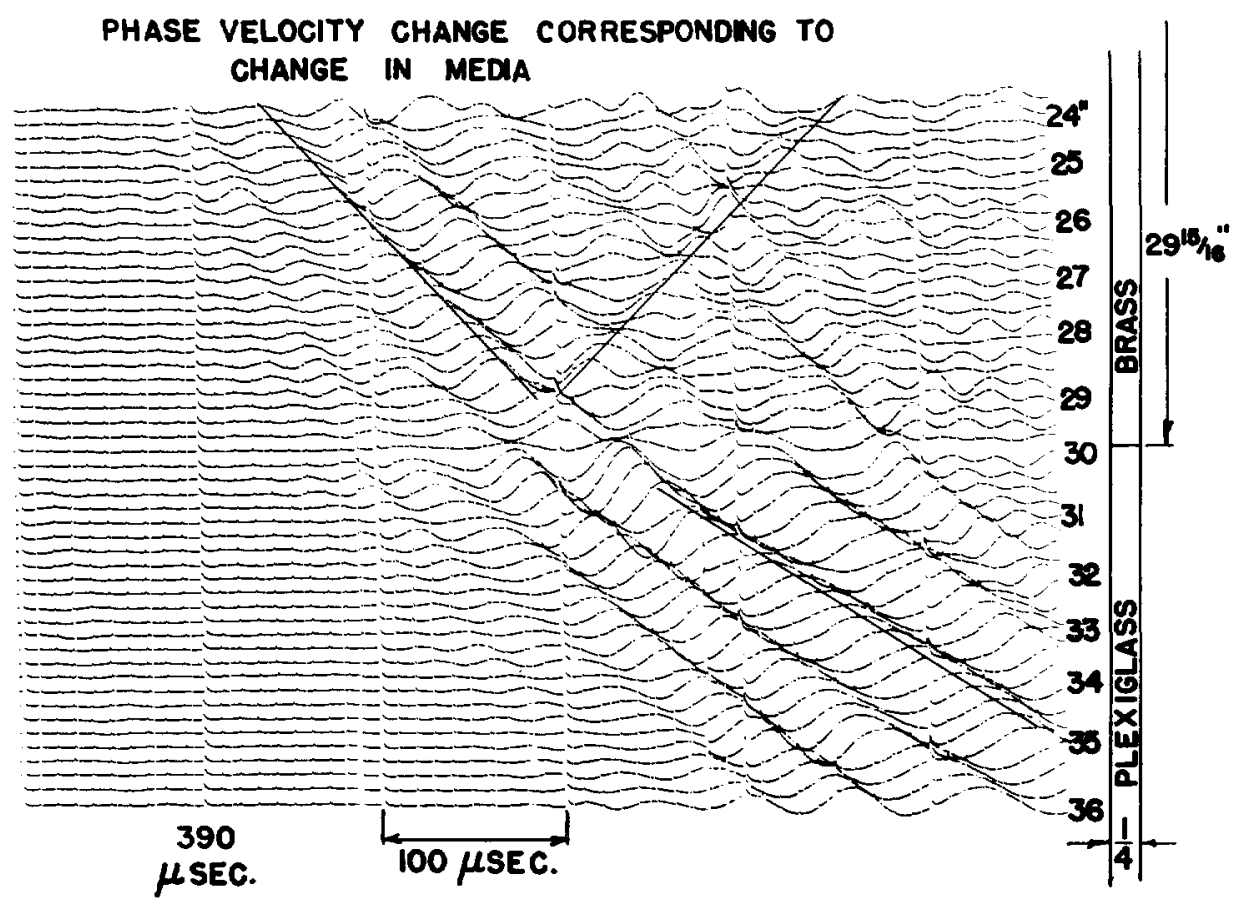

FIG. 5. Model seismogram indicating phase velocity change corresponding to fault. 


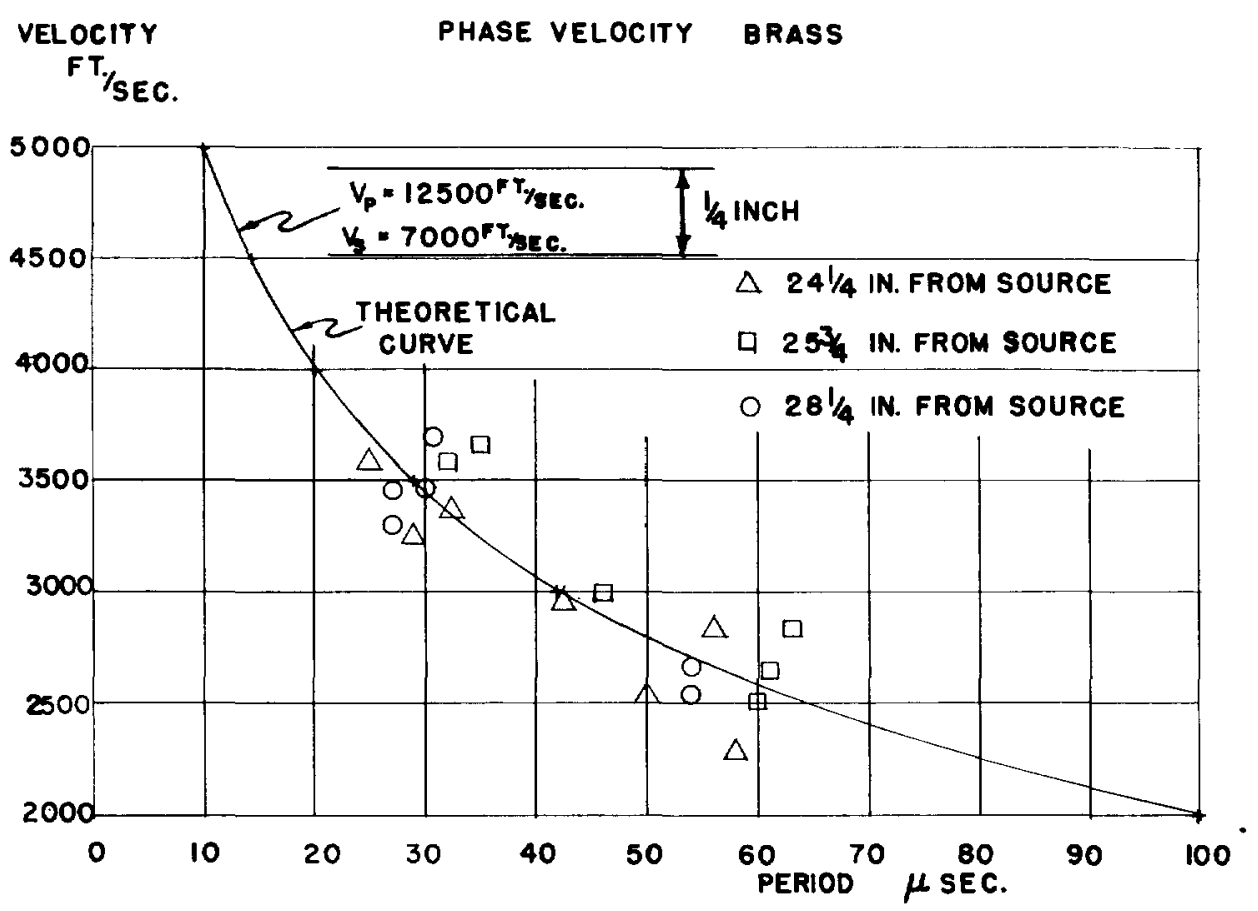

FIG. 6. Comparison of experimental and theoretical phase velocities in brass zone.

abrupt change in phase velocity as the waves cross the fault is apparent. An interesting phenomenon is the occurrence of standing waves and a phase shift in the fault vicinity.

These data will be interpreted by use of theoretical curves. Phase velocities and periods were read directly from the seismograms at several locations in the brass and plexiglass zones. Theoretical curves computed with use of appropriate longitudinal and shear velocities for plexiglass and brass are presented with the experimental data in Figures 6 and 7. The agreement is considered satisfactory and could have been improved by more sophisticated methods of reducing the data, e.g., by the use of machine methods for computing phase shifts for the harmonic components.

Group velocity analysis has been discussed by many investigators (see for example Ewing, Jardetzky, and Press, I957, chapter 4). The usual procedure is to determine an experimental group velocity curve from the overall travel time and distance for each of several periods. The experimental curve is used to select one of many theoretical group velocity curves based on different assumptions of structure. When the path crosses more than one structure, allowance must be made for the group velocity in each structure. This method of analysis is not suitable for local exploration since group velocity determined this way is affected by variations over the entire propagation path. 
A technique used by Brilliant and Ewing (r954) suggests a way in which group velocity methods can be used with greater resolving power. Group velocity is the velocity of propagation of energy of a given frequency, in contrast to phase velocity which is the local velocity of a point of constant phase. Group velocity determined this way does not require knowledge of structures traversed earlier, or of the origin time. It depends on the structure over which the energy velocity measurement is made. Interpretation procedures similar to those described for the phase velocity method may be used as long as the structure is

PHASE VELOCITY

PLEXIGLASS

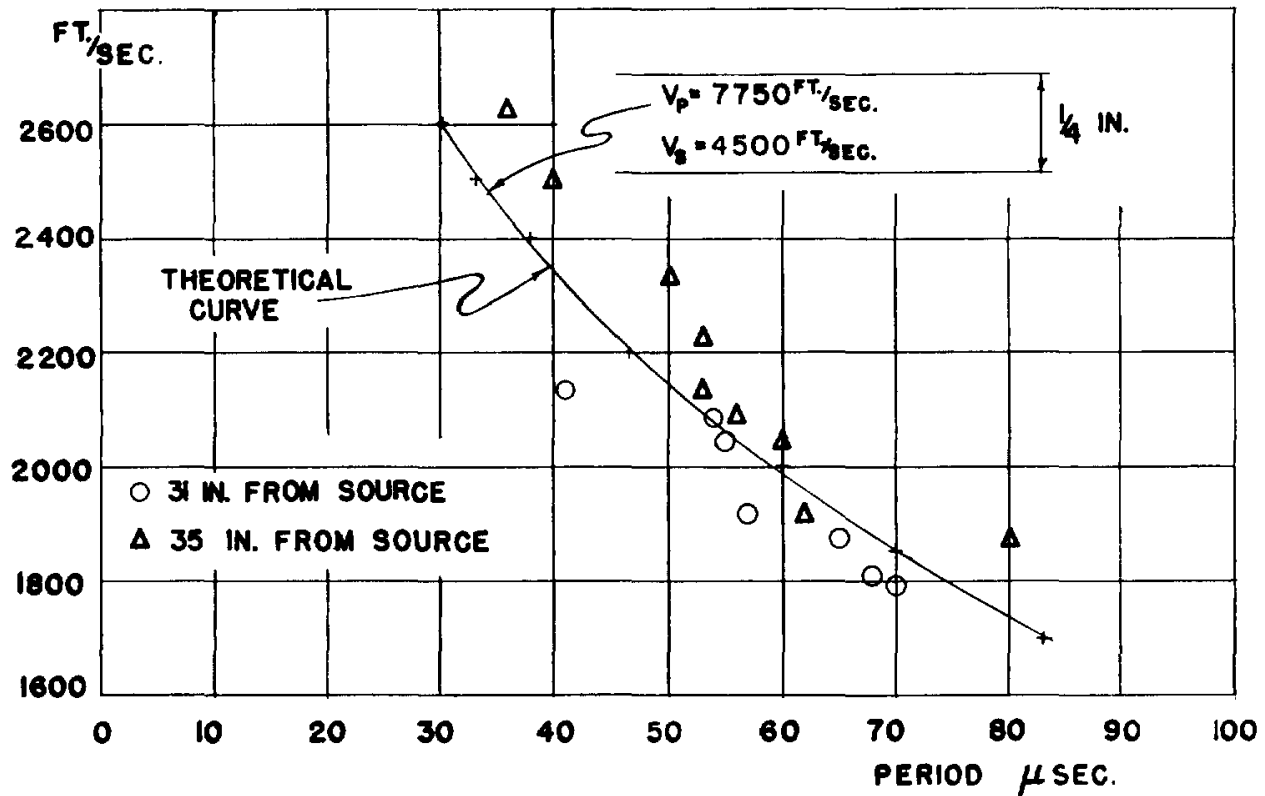

FIG. 7. Comparison of experimental and theoretical phase velocities in plexiglass zone.

homogeneous over the much longer distance required to measure the velocity of a wave group of a given frequency.

As an example, this method will be applied to the fault model. The waves in the plexiglass portion of the seismogram of Figure 5 are assigned numbers. Wave number is plotted as a function of arrival time for several detector positions. By taking slopes of these curves, period as a function of arrival time can be obtained with detector distance as parameter. From these data curves of arrival time versus distance may be constructed for several periods as in Figure 8 . The slopes of the arrival time curves yield group velocity for each of the periods. Experimental group velocity determined in this manner is compared with a theoretical group velocity curve appropriate for a $\frac{1}{4}$ inch plexiglass layer in Figure 9 . 


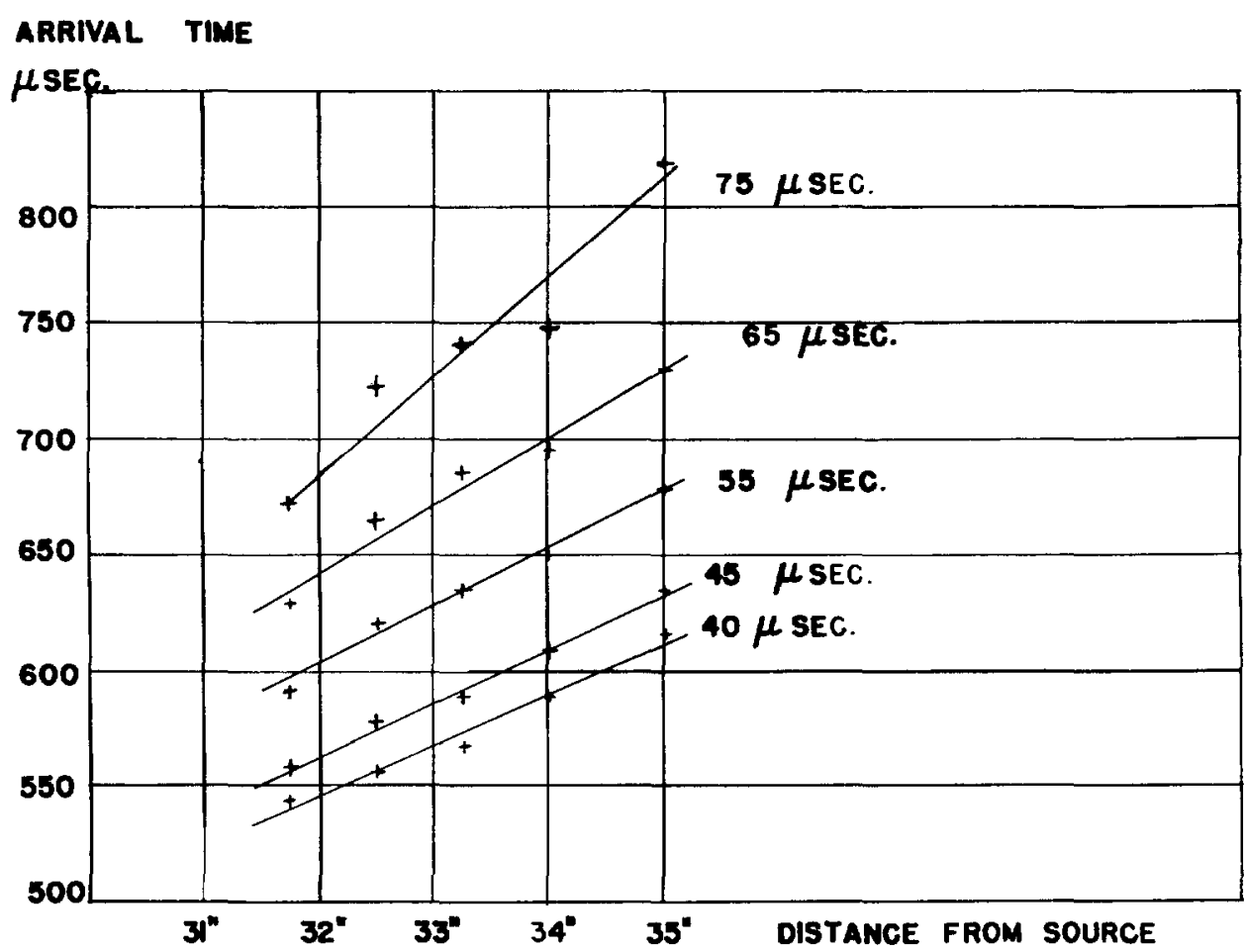

FIG. 8. Travel time curves for several periods in plexiglass zone.

Scarp

Reflected surface waves were indicated in the fault model. The scarp model is presented the better to demonstrate this phenomenon. Reflection of body waves has received much attention. To a lesser degree reflected surface wave pulses have been studied in the ultrasonic scale. The reflection of a train of dispersed surface waves has perhaps received most attention by field seismologists who have observed this phenomenon as returning ground roll. Faults, and topographic changes have been identified as the cause of ground roll reflection.

Reflected flexural waves were excited in a model consisting of an abruptly terminated brass strip (Figure Io). Incident and reflected waves are readily identifiable on the seismogram, and the standing wave pattern formed by their interference is evident. The reflected train is more pronounced in the scarp model than in the fault model as may be seen by comparing Figures 5 and 10 , a result which is not unexpected.

The reflected train is longer, a consequence of dispersion and the accompanying stretching of the wave train with increasing propagation distance. The distance between nodes in the standing wave region is about $\frac{7}{8}$ inches, agreeing with the value for half wavelength of $60 \mu$ s period flexural waves. 


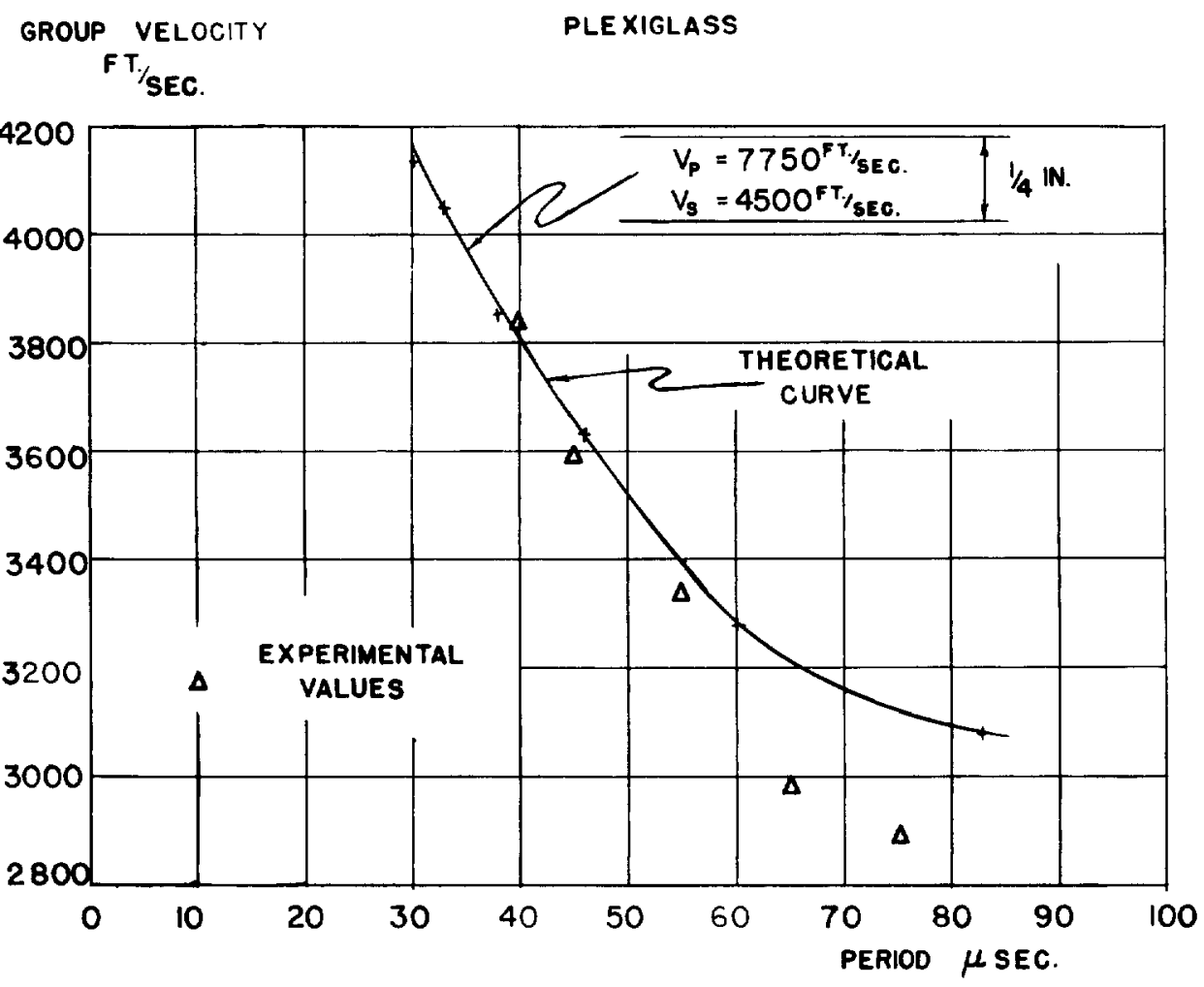

FIG. 9. Comparison of theoretical and experimental group velocity in plexiglass zone.

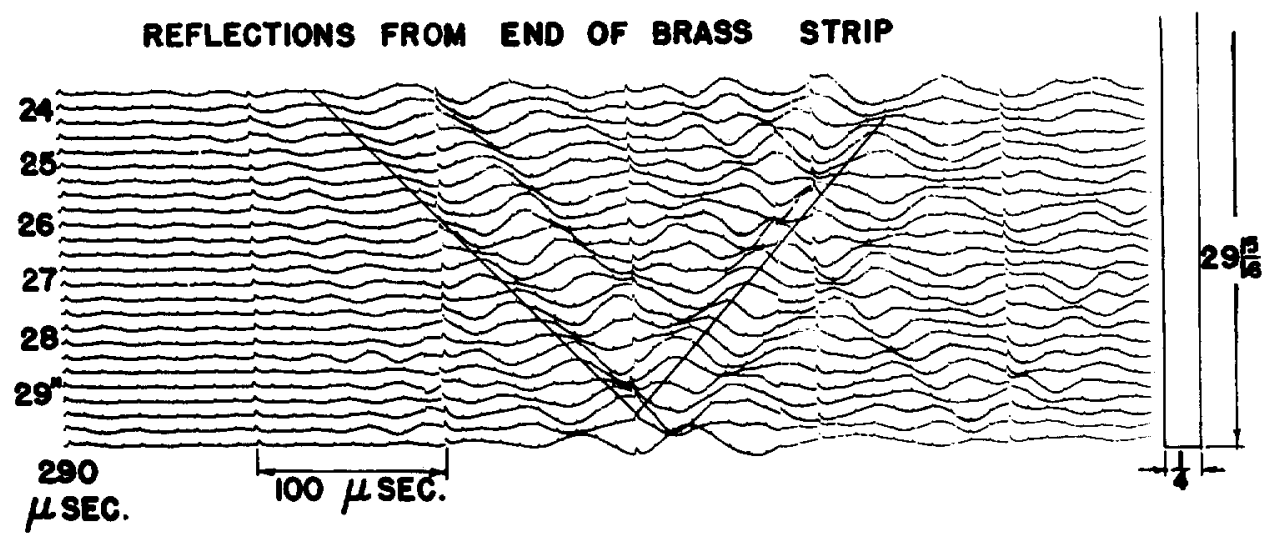

Fig. 1o. Model seismogram of flexural waves reflected from end of a strip (scarp). 


\section{DISCUSSION}

These examples demonstrate how horizontally traveling energy in the form of guided waves can indicate structural changes of the kind encountered by exploration geophysicists. Interpretation of phase velocity changes is not unique in that thickness changes cannot readily be distinguished from lithology changes without additional information. This suggests methods of interpretation involving intercomparison of experimental data rather than the use of theoretical phase velocity curves. Phase velocity anomalies, for example, could play an exploration role similar to gravity anomalies or anomalous leads in fan shooting methods. A further analogy with gravity is the effect of an entire section on phase velocity in contrast to mapping of single beds by refraction and reflection techniques. Thus overall changes in section such as would be produced by salt domes, porosity and lithology changes, basement highs, reefs, etc., might be examined by phase velocity methods.

To apply these methods in field exploration, one would have to use guided waves in the frequency range $\mathbf{I}$ to $\mathrm{I} / \mathrm{I} O \mathrm{cps}$. These low frequencies are required in order to obtain penetration to depths of interest in exploration. There is no doubt that waves in this frequency range occur in nature. Earthquake surface waves are an example of waves at one end of this band whereas ground roll limits the other end.

In view of the success of the method when used in crustal studies and model studies, a field research program seems warranted. This program should concern itself with methods of excitation of guided waves in the frequency band of interest, methods of recording these waves and computing their phase velocity automatically, and methods of interpretation.

\section{REFERENCES}

Brilliant, R., and M. Ewing, I954, Dispersion of Rayleigh waves across the U. S.: Seismol. Soc. Amer. Bull., v. 44, P. $149^{-1} 5^{8}$.

Bullen, K. E., I953, An introduction to the theory of seismology, University Press, Cambridge, $296 \mathrm{p}$.

Evernden, J., I953, Direction of approach of Rayleigh waves and related problems (Part I): Seismol. Soc. Amer. Bull., v. 44 , p. $159^{-1} 84$.

Ewing, M., and F. Press, 1956, Surface waves and guided waves: Encyclopedia of Physics, v. 47, p. II9-I 39 .

Ewing, M., Jardetzky, W., and F. Press, 1957, Propagation of elastic waves in layered media: New York, McGraw-Hill Book Co.

Oliver, J., Press, F., and M. Ewing, r954, Two-dimensional model seismology: Geophysics, v. I9, p. 202-21g.

Press, F., I956, Determination of crustal structure from phase velocity of Rayleigh waves. Part I, Southern California: Geol, Soc. Amer. Bull., v. 67, p. 1647-1658.

Satô, Y., I95r, Study in surface waves. II: Bull. Earthquake Research Institute (Tokyo), v. 29, p. 223-262. 